Cite this: Chem. Sci., 2019, 10, 239

๑ All publication charges for this article have been paid for by the Royal Society of Chemistry

Received 6th March 2018

Accepted 3rd October 2018

DOI: $10.1039 / c 8 s c 01074 a$

rsc.li/chemical-science
Check for updates

\section{A functional interplay between intein and extein sequences in protein splicing compensates for the essential block B histidine $\uparrow$}

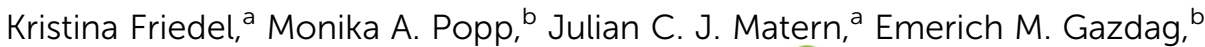 \\ Ilka V. Thiel, ${ }^{a}$ Gerrit Volkmann, ${ }^{a}$ Wulf Blankenfeldt (iD bc and Henning D. Mootz (DD *a
}

Inteins remove themselves from a precursor protein by protein splicing. Due to the concomitant structural changes of the host protein, this self-processing reaction has enabled many applications in protein biotechnology and chemical biology. We show that the evolved M86 mutant of the Ssp DnaB intein displays a significantly improved tolerance towards non-native amino acids at the $\mathrm{N}$-terminally flanking (-1) extein position compared to the parent intein, in the form of both an artificially trans-splicing split intein and the cis-splicing mini-intein. Surprisingly, side chains with increased steric bulk compared to the native $G$ ly(-1) residue, including D-amino acids, were found to compensate for the essential block B histidine in His73Ala mutants in the initial $\mathrm{N}-\mathrm{S}$ acyl shift of the protein splicing pathway. In the case of the M86 intein, large $(-1)$ side chains can even rescue protein splicing activity as a whole. With the comparison of three crystal structures, namely of the M86 intein as well as of its Gly(-1)Phe and Gly(-1) Phe/His73Ala mutants, our data supports a model in which the intein's active site can exert a strain by varying mechanisms on the different angles of the scissile bond at the extein-intein junction to effect a ground-state destabilization. The compensatory mechanism of the block B histidine is the first example for the direct functional role of an extein residue in protein splicing. It sheds new light on the exteinintein interplay and on possible consequences of their co-evolution as well as on the laboratory engineering of improved inteins.

\section{Introduction}

Inteins are protein elements that remove themselves from their precursor proteins in an autocatalytic pathway called protein splicing. ${ }^{1-3}$ The mature host protein is produced by the ligation of the flanking sequences, referred to as $\mathrm{N}$ - and C-terminal exteins, with a native peptide bond. The posttranslational alteration of the peptide backbone and hence of the protein's structure has enabled a plethora of applications, many of which involve mutated or otherwise engineered inteins to control the protein splicing pathway or induce off-pathway cleavage reactions. Protein splicing is a multi-step process of intramolecular rearrangements that keeps the intein and extein segments covalently attached until the exteins are connected (Fig. 1A)..$^{3-5}$ In the first step of the canonical class 1 inteins, ${ }^{6}$ the scissile

${ }^{a}$ Institute of Biochemistry, University of Muenster, Wilhelm-Klemm-Str. 2, 48149 Münster, Germany. E-mail: Henning.Mootz@uni-muenster.de

${ }^{b}$ Structure and Function of Proteins, Helmholtz Centre for Infection Research, Inhoffenstraße 7, 38124, Braunschweig, Germany

'Institute for Biochemistry, Biotechnology and Bioinformatics, Technische Universität Braunschweig, Spielmannstraße 7, 38106 Braunschweig, Germany

$\dagger$ Electronic supplementary information (ESI) available. See DOI: $10.1039 / \mathrm{c} 8 \mathrm{sc} 01074 \mathrm{a}$ peptide bond upstream of the intein rearranges in an $\mathrm{N}-\mathrm{S}$ (or $\mathrm{N}-\mathrm{O}$ ) acyl shift involving the cysteine (or serine) side chain as the first amino acid of the intein (residue 1) and the upstream flanking amino acid of the $\mathrm{N}$-extein (residue $(-1)$ ). The resulting linear thioester (or oxoester) is then transesterified onto the sulfhydryl (or hydroxyl) side chain of a cysteine (or serine/ threonine) as the first amino acid downstream of the intein, the $(+1)$ residue, to give a branched intermediate. In the third step catalyzed by the intein, the asparagine (or glutamine) side chain preceding the C-terminal scissile bond attacks the downstream intein-extein junction to effect its cleavage with concomitant cyclization of the side chain. Finally, the thioester (or oxoester) bond connecting the $\mathrm{N}$ - and C-terminal exteins rearranges in an uncatalyzed $\mathrm{S}-\mathrm{N}$ (or $\mathrm{O}-\mathrm{N}$ ) acyl shift to give a stable peptide bond in the mature splice product (Fig. 1A).

Inteins can be referred to as single-turnover enzymes because they employ typical enzymatic strategies to bring about the reactions. However, they do catalyze the reaction only once. Mutational studies on several inteins have led to the idea that inteins use varying strategies to catalyze the individual steps. In particular, conserved residues from conserved block motifs may contribute to a different extent when comparing different inteins. For example, in the case of the initial reaction, the N-S (or N-O) acyl shift, a histidine from the block B motif, also 
A

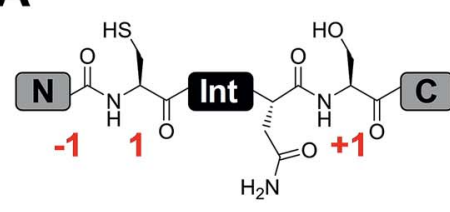

B<smiles>NC(=O)C[C@@H](C=C[C@@H](N)CS)C(=O)N[C@@H](CO)C(=O)CC(F)(F)F</smiles>

C

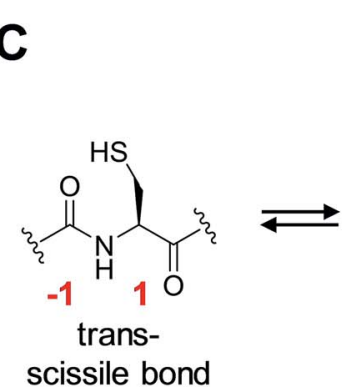

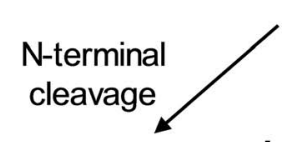

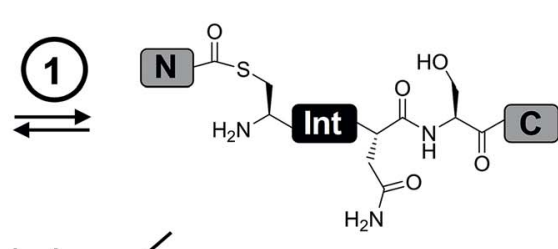

$\mid$
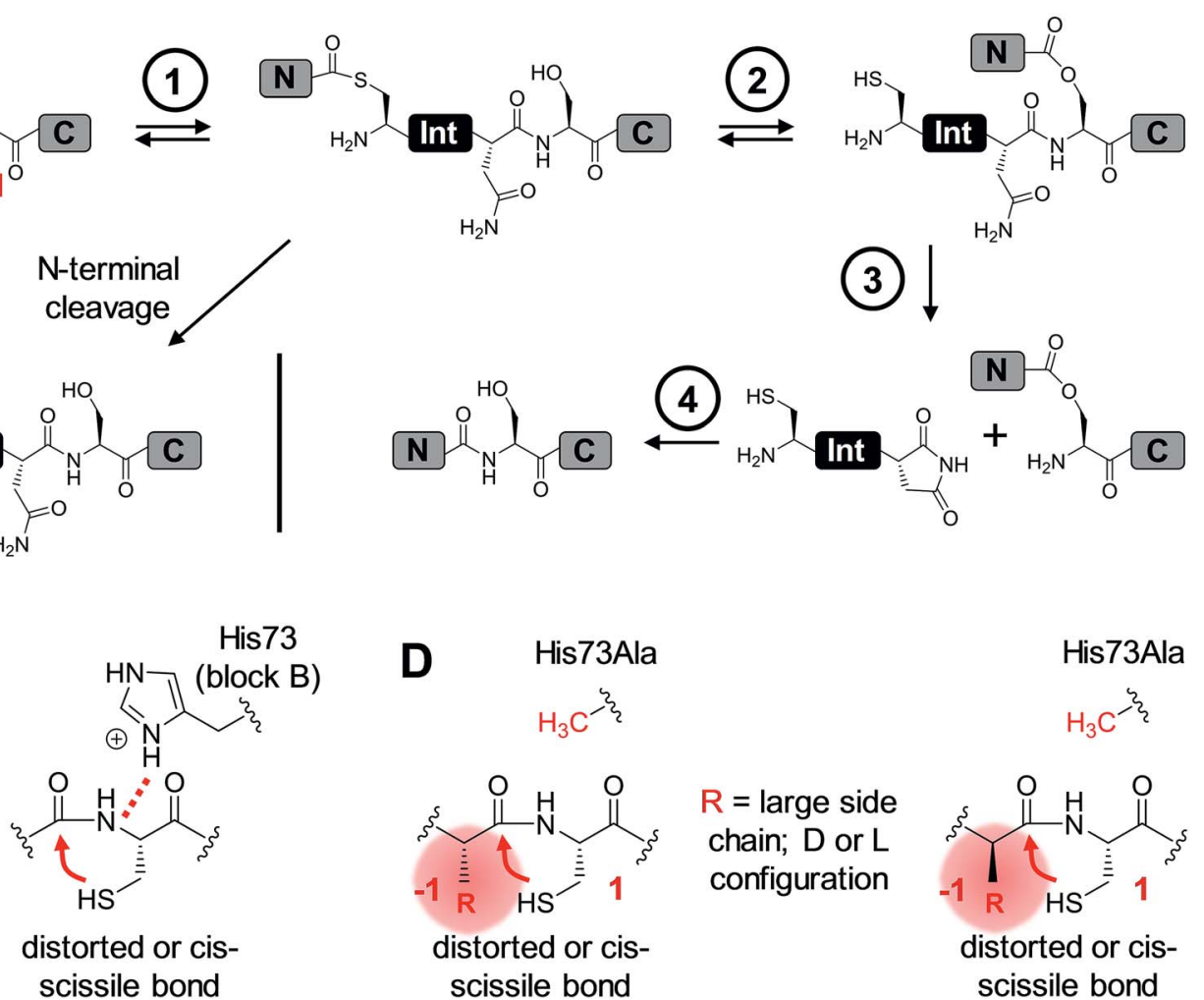

$R=$ large side
chain; $D$ or $L$
configuration

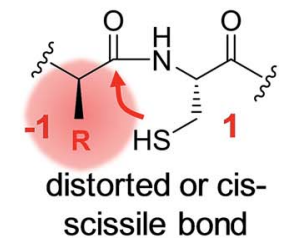

Fig. 1 Mechanisms of protein splicing and the N-S acyl shift. (A) Mechanism of the protein splicing pathway of class 1 inteins. Shown here are Cys and Ser residues at the 1 and +1 positions, respectively, as present in the Ssp DnaB intein. Step 1: N-S acyl shift to the linear thioester intermediate; step 2: trans-esterification to the branched intermediate; step 3: asparagine cyclization to effect succinimide formation and cleavage of the $\mathrm{C}$-terminal scissile bond; step 4: spontaneous $\mathrm{O}-\mathrm{N}$ shift to the final splice product (uncatalyzed). (B) Side-reaction of $\mathrm{N}$-terminal cleavage through nucleophilic attack of the linear thioester. This step is enforced when the C-terminal splice junction is mutated. (C) Mechanistic contribution of the essential block B histidine in the $\mathrm{N}-\mathrm{S}$ acyl shift. (D) New artificial mechanisms to compensate for a lacking block B histidine as proposed in this work. Red numbers indicate the nomenclature in residue numbering at the extein-intein junctions.

referred to as the N3 motif, ${ }^{7}$ is the single most highly conserved amino acid in inteins. It is believed to polarize the N-terminal scissile bond and thereby effect a ground-state destabilization (Fig. 1C), ${ }^{8-11}$ although other studies have suggested roles in deprotonation of the Cys1 side chain or the stabilization of the oxyanion of the tetrahedral intermediate. ${ }^{12}$ The block B histidine is essential in many inteins including the Ssp DnaB intein. ${ }^{\mathbf{1 0 , 1 3 , 1 4}}$ However, in others, such as the Pab PolII intein, it is only important, i.e., its mutation lowers but does not fully impair the efficiency of protein splicing. ${ }^{15}$ There is even a very small subset of inteins that lack the histidine side chain. In this case another residue, which is not important in inteins containing the block B histidine, acts in a compensatory mechanism, presumably by stabilizing the tetrahedral intermediate. ${ }^{16}$ Such a flexible catalysis might have been allowed during evolution because no repeated catalytic turnover is required and the selective pressure on a highly optimized catalytic strategy likely was limited. ${ }^{16}$

The initial $\mathrm{N}-\mathrm{S}$ acyl shift in protein splicing is also of great interest in the field of chemical ligation by native chemical ligation or expressed protein ligation because a peptide or protein thioester is required here and interception of the protein splicing pathway is the method of choice for making C-terminal thioesters of recombinant proteins. ${ }^{17,18}$ Chemical strategies to facilitate acyl shift reactions for the preparation of thioester peptides include the use of an $N$-alkylated cysteine. ${ }^{19-22}$ An $N$-methyl cysteine in the active site of the $S s p$ DnaB intein was found to functionally compensate a block B histidine mutant. ${ }^{10}$

In this work, we have studied the extein sequence dependence of the Ssp DnaB intein ${ }^{23}$ and its evolved M86 mutant, ${ }^{24}$ both in trans- and in cis-splicing forms. ${ }^{25,26}$ By serendipity, we discovered a compensatory mechanism for the loss of the block B histidine that is dependent on the size of the upstream flanking $(-1)$ residue. Crystal structures of an M86 precursor intein as well as of two extein mutants with and without the block B histidine help explain the compensatory mechanism by implying steric effects to distort the $\mathrm{N}$-terminal splice junction.

\section{Results and discussion}

The M86 mutant intein displays increased splicing promiscuity with regard to the upstream flanking amino acid

The M86 mutant was previously selected by sequential directed evolution from the parent $S s p$ DnaB intein ${ }^{23}$ as a more general protein splicing catalyst with regard to the flanking amino acids, a highly desirable trait when choosing a ligation site. ${ }^{24}$ Indeed, several studies indicated its increased sequence 
promiscuity, both in the form of the original mini-intein as well as an engineered split intein. In the latter case, an N-terminal fragment $\left(\mathrm{Int}^{\mathrm{N}}\right)$ of 11 amino acids and a C-terminal fragment $\left(\mathrm{Int}^{\mathrm{C}}\right.$ ) of 143 amino acids were generated. ${ }^{24,27-29}$ However, a systematic analysis of individual substitutions of the extein amino acids immediately flanking the intein has not been reported yet. To this end, we decided to study the tolerance towards the $(-1)$ residue at the position upstream of the N-terminal scissile bond. We compared the parent $S s p$ DnaB intein and the M86 mutant in the context of the semi-synthetic split inteins. A set of synthetic $\mathrm{Ex}^{\mathrm{N}}-\mathrm{Int}^{\mathrm{N}}$ peptides pep1 to pep9 was synthesized with various residues of increasing steric bulk at the $(-1)$ extein position, ranging from Gly as observed in the wild-type intein to large side chains and amino acids in the D-configuration (Table 1). 5,6-Carboxyfluorescein (Fl-) was included in the short extein ${ }^{\mathrm{N}}$ sequence to facilitate analysis of the splicing and cleavage reactions. Note that all 8 mutations of the M86 intein are located in the $\mathrm{Int}^{\mathrm{C}}$ fragment. Whereas the parent wild-type intein $\left(\mathrm{WT} \operatorname{Int}^{\mathrm{C}}\right.$ ) with thioredoxin (Trx) as the model extein ${ }^{\mathrm{C}}$ sequence was able to efficiently splice only with the native $\operatorname{Gly}(-1)$ residue, the corresponding M86 construct $\left(\mathrm{M} 86 \mathrm{Int}^{\mathrm{C}}\right.$ ) exhibited robust activity in all cases (Fig. 2 and Table 1). Table S1 and Fig. S1A $\uparrow$ show the yields and the kinetic constants for these trans-splicing reactions. Additional fractions of the Int ${ }^{\mathrm{C}}$ partner proteins were converted into the C-terminal cleavage products by breaking of the scissile bond downstream of the intein through cyclization of the catalytic block $G$ asparagine residue, indicating that complex formation of the intein fragments had occurred but resulted in the off-pathway reaction (Fig. 2). Together, these findings underlined the improved sequence tolerance of the M86 mutant.

Large side chains at position (-1) can compensate for a lack of the catalytic block B histidine in the N-S acyl shift

We aimed to study the association step of the intein fragments to complex formation. ${ }^{24,30}$ To this end, we incubated the Int $^{\mathrm{N}}$ peptides with triple mutants of the WT Int ${ }^{\mathrm{C}}$ and M86 $\mathrm{Int}^{\mathrm{C}}$ constructs, designed to be inactive in all three steps of the protein splicing pathway $(\mathrm{H} 73 \mathrm{~A} / \mathrm{N} 154 \mathrm{~A} / \mathrm{S}(+1) \mathrm{A}$, referred to as WT $\operatorname{Int}^{\mathrm{C}}(\mathrm{AAA})$ and M86 $\mathrm{Int}^{\mathrm{C}}(\mathrm{AAA})$ ), to form complex I (Fig. 3A).
Note that the non-covalent complex between WT Int ${ }^{\mathrm{C}}$ (AAA) and pep1 $\left(\right.$ Int $^{\mathrm{N}}$ peptide with $\mathrm{Gly}(-1)$ ) could be observed on a Coomassie-stained denaturing SDS gel when the samples had not been boiled. The fluorescence of this complex results from the carboxyfluorescein moiety in the $\mathrm{Ex}^{\mathrm{N}}$ portion of the intact pep1, as reported previously (detected under UV illumination of the gel, see second lane in lower panel in Fig. 3B). ${ }^{30}$ Surprisingly, a loss of fluorescence in the complex band was observed for all residues except the native $\mathrm{Gly}(-1)$ (pep1) in the case of the WT intein and for all larger side chains at the $(-1)$ position (pep5 to pep9) in the case of the M86 intein (Fig. 3B, lower panels). Loss of the fluorophore by $\mathrm{N}$-terminal cleavage indicated that first the peptide bond must have rearranged into the thioester, which then underwent cleavage by hydrolysis or thiolysis with DTT according to the scheme in Fig. 1B. Comparison with the band for the complex on the Coomassie-stained SDS gel indicated different yields of complex formation. In the case of the WT intein, with Ala, Abu and Thr at the flanking $(-1)$ position (pep2 to pep4), the complexes seemed stable only to a low degree under the conditions of the SDS gel and only marginal fluorescence could be detected, possibly reflecting the lower complex yield. Peptides pep5 to pep9 harboring larger side chains at the $(-1)$ position showed higher amounts of stable complexes; however, these were also not or only marginally fluorescent, indicating a loss of the $\mathrm{Ex}^{\mathrm{N}}$ parts with the fluorophore (Fig. 3B). In the case of the M86 intein, stable complexes were observed for all peptides at nearly quantitative yields (Fig. 3B). While the assays with peptides containing Gly (pep1), Ala (pep2), or Abu (pep3) at the $(-1)$ position yielded highly fluorescent complexes, the fluorescent signal was mostly or even nearly completely lost in the case of the larger amino acids Leu (pep5), His (pep6), Phe (pep7), D-Leu (pep8), and D-Phe (pep9) at the $(-1)$ position. The peptide with $\operatorname{Thr}(-1)$ (pep4) resulted in a phenotype between these two groups. Indeed, the assay with pep4 revealed a distinct double band for the intein complex in the Coomassie-stained gel, but not under UV illumination, indicating that two different complexes were present and could be separated on the gel (Fig. 3B). A cleavage of the fluorescent $\mathrm{Ex}^{\mathrm{N}}$ also results in the liberated $\mathrm{Int}^{\mathrm{N}}$ part. We therefore assigned the two complexes of the $\operatorname{Int}^{\mathrm{C}}(\mathrm{AAA})$ proteins to one with an $\mathrm{Ex}^{\mathrm{N}}-\mathrm{Int}^{\mathrm{N}}$ peptide (complex I, fluorescent) and

Table 1 List of $\operatorname{Int}^{\mathrm{N}}$ peptides used in this study

\begin{tabular}{|c|c|c|c|c|c|c|c|}
\hline Peptide no. & Sequence $^{a}$ & \multicolumn{2}{|c|}{ Splicing $^{b}[\%]$} & \multicolumn{2}{|c|}{$\mathrm{N}$-terminal cleavage ${ }^{c}[\%]$} & \multicolumn{2}{|c|}{ Splicing $^{b}[\%]$} \\
\hline pep2 & Fl-KKESA-Int ${ }^{\mathrm{N}}$ & 2 & $63 \pm 1$ & $9 \pm 2$ & $7 \pm 2$ & 0 & 3 \\
\hline pep3 & Fl-KKESAbu-Int ${ }^{\mathrm{N}}$ & 1 & $61 \pm 2$ & $18 \pm 2$ & $19 \pm 3$ & 0 & 4 \\
\hline pep4 & Fl-KKEST-Int ${ }^{N}$ & 0 & $37 \pm 3$ & $31 \pm 2$ & $53 \pm 4$ & 0 & $21 \pm 1$ \\
\hline pep7 & Fl-KKESF-Int ${ }^{N}$ & 0 & $44 \pm 2$ & $75 \pm 2$ & $90 \pm 2$ & 0 & $22 \pm 3$ \\
\hline pep8 & Fl-KKESDL-Int ${ }^{\mathrm{N}}$ & 0 & $42 \pm 1$ & $69 \pm 4$ & $100 \pm 0$ & 0 & 3 \\
\hline pep9 & Fl-KKESDF-Int ${ }^{N}$ & 0 & $45 \pm 0$ & $100 \pm 0$ & $100 \pm 0$ & 0 & $17 \pm 5$ \\
\hline
\end{tabular}

${ }^{a}$ Int $^{\mathrm{N}}=$ CISGDSLISLA, $\mathrm{Fl}=5$,6-carboxyfluoresceine. ${ }^{b}$ Determined by SDS-PAGE analysis. ${ }^{c}$ Determined by HPLC analysis. 

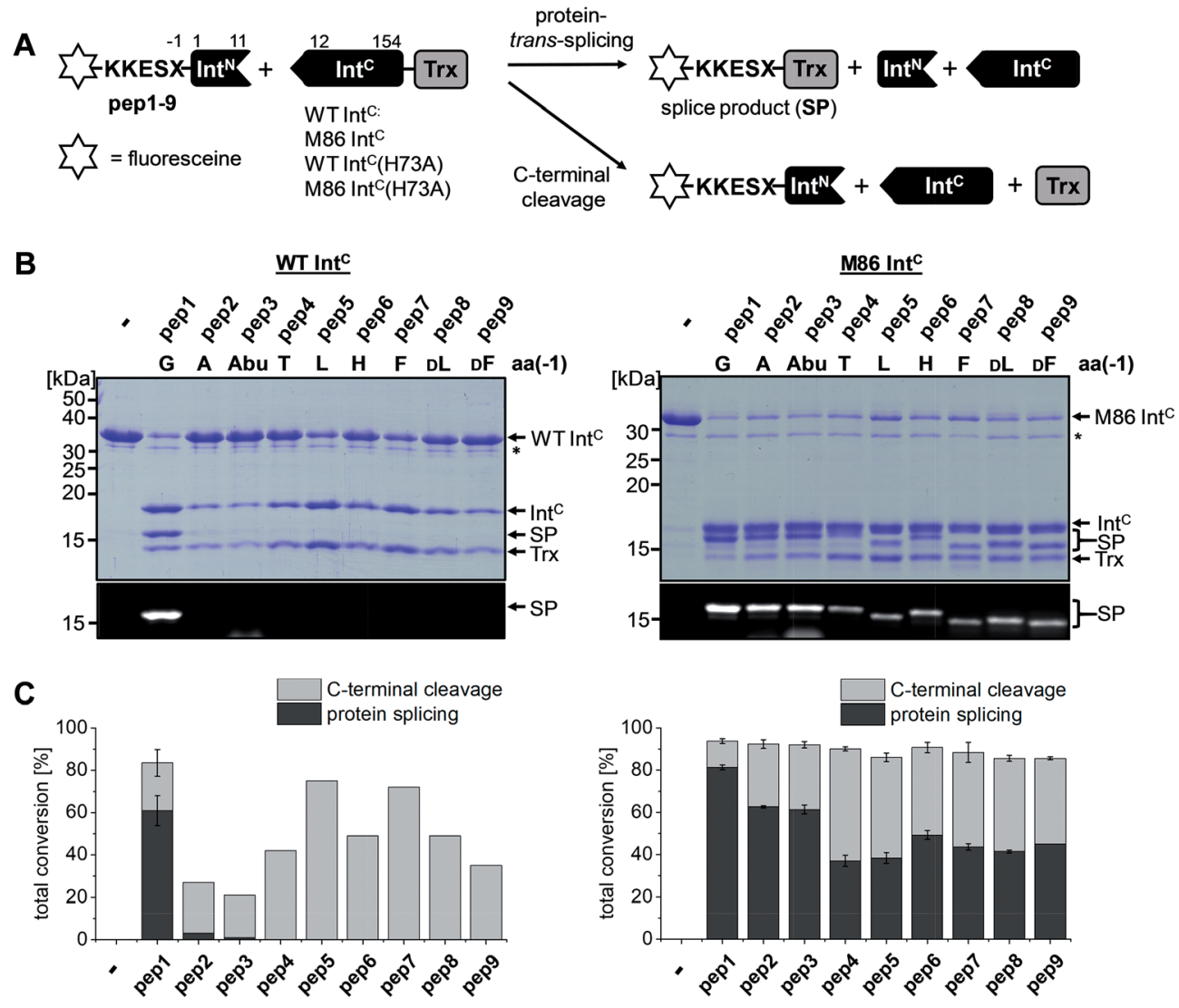

Fig. 2 Extein dependence at the (-1) position of semisynthetic protein trans-splicing. (A) Scheme of the reactions involving the WT Ssp DnaB and M86 inteins. (B) Analysis of reactions on SDS-PAGE gels using Coomassie-staining (top) or UV illumination (bottom panel). Int ${ }^{C}$ proteins (20 $\mu M$ ) were incubated with the indicated peptides $(60 \mu \mathrm{M})$ for $24 \mathrm{~h}$ and then quenched by SDS loading buffer and boiling. The variation of a single residue had a surprisingly large impact on the migration behavior of some of the splice products (SP). The weak bands visible for pep3 in the lower panels seem to result from a slight impurity of this peptide and were also observed in the absence of a protein partner (data not shown). (C) Yields of protein trans-splicing and C-terminal cleavage reactions determined by densitometric analysis of the Coomassie-stained gels shown in B. Error bars indicate standard deviations. Asterisks denote a protein contamination.

another with the remaining Int $^{\mathrm{N}}$ peptide (complex II, nonfluorescent). We reasoned that separation of the two complexes on the SDS-gel is possible in some cases but not necessarily in all cases.

These results suggested that for the M86 intein both types of complexes were stable under the SDS conditions. In contrast, for the WT intein the complexes appeared less stable. Our observations would be consistent with all $(-1)$ residues larger than the native $\mathrm{Gly}(-1)$ rendering the $\mathrm{Ex}^{\mathrm{N}}-\mathrm{Int}^{\mathrm{N}}$ complexes unstable (complex I) under these conditions and that the Coomassie-stained SDS gels only showed the cleaved complexes with the Int ${ }^{\mathrm{N}}$ peptide (complex II). These differences in complex stability would be in agreement both with the higher fragment affinity of the M86 mutant in the case of the split intein $\mathbf{s}^{24}$ and with the higher thermostability of the cis-M86 intein compared to that of the cis-WT intein. ${ }^{31}$ However, since the SDS-PAGE based assays shown in Fig. 3 cannot be analyzed with sufficient quantitative accuracy, we developed an HPLC-assay to directly show and quantify the $\mathrm{N}$-terminal cleavage by detecting the $\mathrm{Ex}^{\mathrm{N}}$ parts (Fig. 4). While pep1 with $\mathrm{Gly}(-1)$ was completely stable upon incubation with the $\operatorname{Int}^{\mathrm{C}}$ partners WT $\operatorname{Int}^{\mathrm{C}}(\mathrm{AAA})$ and M86 $\operatorname{Int}^{\mathrm{C}}(\mathrm{AAA})$, peptides with larger side chains at the $(-1)$ position were cleaved at increasingly higher levels up to virtual completion (see Table 1 for quantification). These findings were consistent with our interpretation of the SDS-PAGE data. They confirmed that the block B histidine is essential for the $\mathrm{N}-\mathrm{S}$ acyl shift with the native Gly $(-1)$ residue; however, it was functionally compensated for by larger side chains at the $(-1)$ position, in particular by those larger than Abu, both for the WT and the M86 inteins.

The compensatory mechanism for the $\mathrm{N}-\mathrm{S}$ acyl shift supports protein splicing in a block $\mathrm{B}$ histidine mutant

We next asked whether the unexpected compensatory mechanism for the otherwise essential role of the block B histidine functions only for the isolated N-S acyl shift or could also rescue the entire process of protein trans-splicing. This question is important as the observed effect could have been accidentally favored by a combination of the three active site substitutions in the triple mutants of the WT and M86 $\mathrm{Int}^{\mathrm{C}}$ parts used to study the isolated $\mathrm{N}-\mathrm{S}$ acyl shift. As expected, no splicing activity above marginal background levels was observed for the H73A single mutant of the WT intein with its native Gly $(-1)$ residue of 


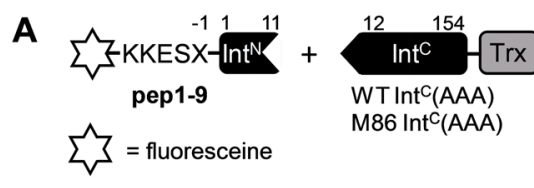

B

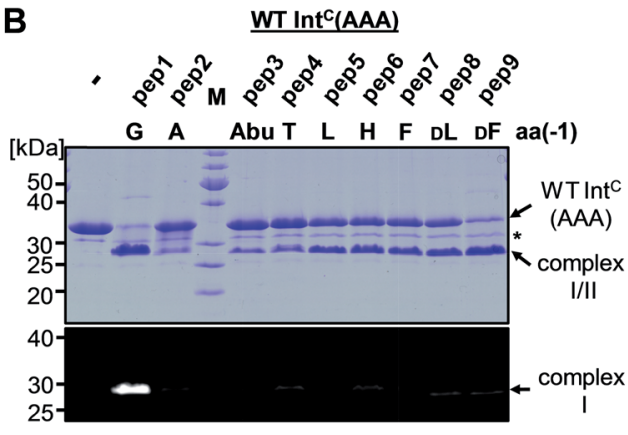

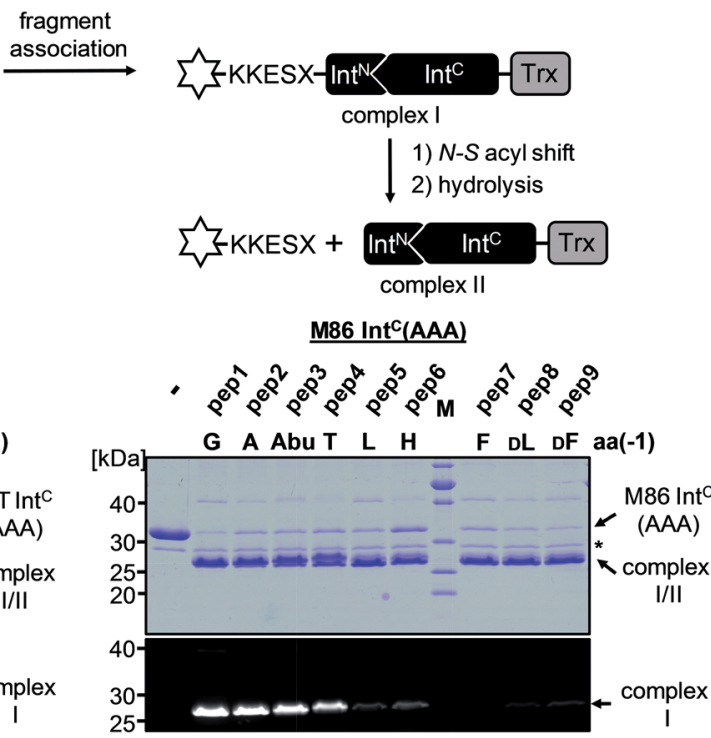

Fig. 3 Complex formation and N-terminal cleavage of split intein pairs blocked in the essential residues of the block B and G motifs. (A) Scheme of the reactions involving the triple mutants (H73A, N154A, S+1A) of the WT Ssp DnaB and M86 inteins. (B) Analysis of reactions on SDS-PAGE gels using Coomassie-staining (top) or UV illumination (bottom panel). Int ${ }^{C}$ proteins $(20 \mu \mathrm{M})$ were incubated with the indicated peptides $(60 \mu \mathrm{M})$ for $24 \mathrm{~h}$ and then quenched by SDS loading buffer without boiling. Asterisks denote a protein contamination.

A

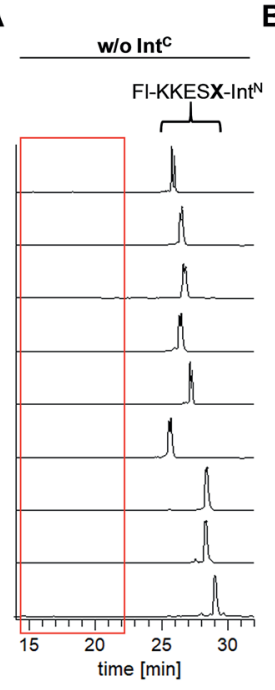

B

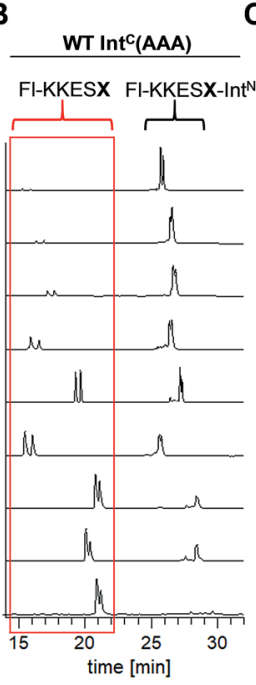

C

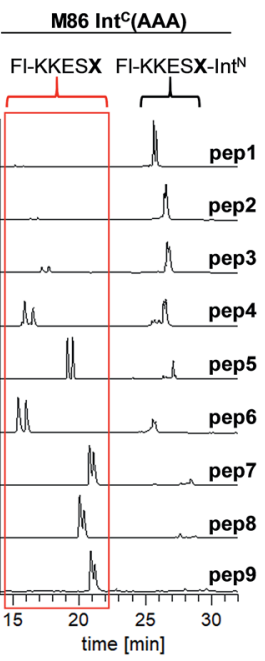

Fig. 4 HPLC analysis of N-terminal cleavage with $\operatorname{Int}^{\mathrm{C}}$ constructs containing triple mutations in the blocks $\mathrm{B}$ and $\mathrm{G}$. Analyses of the $\mathrm{Ex}^{\mathrm{N}}-$ Int ${ }^{\mathrm{N}}$ peptides (Fl-KKESX-Int ${ }^{\mathrm{N}}$ ) are shown before (left panel) and after (middle and right panels) incubation with $\mathrm{Int}^{\mathrm{C}}$ constructs. Absorption was measured at $280 \mathrm{~nm}$, which detects only the Ex ${ }^{\mathrm{N}}$ portion of the peptides (Fl-KKESX), but not the cleaved $\operatorname{Int}^{\mathrm{N}}$ portion. $\operatorname{Int}^{\mathrm{C}}$ proteins $(15 \mu \mathrm{M})$ and peptides $(30 \mu \mathrm{M})$ were incubated for $24 \mathrm{~h}$. Samples were acidified with $0.1 \%$ TFA and boiled for $\operatorname{Int}^{C}$ precipitation prior to HPLC analysis. Note that the double peaks originate from the 5,6-isomers of the carboxyfluoresceine moiety. The identities of the cleaved Ex ${ }^{\mathrm{N}}$ fragments were verified by MS-analysis (data not shown). The percentages of $\mathrm{N}$-terminal cleavage are given in Table 1 . The red boxes highlight the $\mathrm{N}$-terminal cleavage products.

pep1. Also the other peptides with bulkier amino acids at the $(-1)$ position showed only marginal yields (Fig. 5). However, large amino acids at the $(-1)$ position did enable protein trans-

splicing with the H73A single mutant of the M86 intein, reaching significant efficiencies of $\sim 20-30 \%$ yield (Fig. 5 and Table 1). Interestingly, the same side chain dependence as for the isolated $\mathrm{N}-\mathrm{S}$ acyl shift was observed as splicing occurred only with $(-1)$ residues larger than Abu. The only exception was pep8 with D-Leu at position $(-1)$, which showed no splicing, whereas pep9 with D-Phe was active. No protein trans-splicing could be detected with the smaller side chains, including the native $\operatorname{Gly}(-1)$ in pep1 (Fig. 5). These observations further underlined our conclusion that an alternative mechanism for the $\mathrm{N}-\mathrm{S}$ acyl shift, dependent on a bulky $(-1)$ residue, must have been at play, and showed that it was compatible with the subsequent steps of protein trans-splicing.

\section{Cis-splicing inteins show a similar effect in response to $(-1)$ mutations as the trans-splicing inteins}

We sought to rule out potential artefacts in the functional behavior of the mutated inteins from the association step of the artificially generated fragments. As a further control, we therefore tested the genetically accessible substitutions at the $(-1)$ position in cis-variants of the WT and M86 mini-inteins. In contrast to the split WT intein, the cis-WT intein displayed some splicing activity with $\mathrm{Ala}(-1)$ and $\operatorname{His}(-1)$, as well as slight activity with $\operatorname{Thr}(-1)$ and Phe(-1) (Fig. 6), indeed suggesting a somewhat more general splice profile of the cis-intein that would be consistent with a more robust protein folding compared to the split intein. Nevertheless, with the exception of the $\operatorname{Gly}(-1)$ construct, large quantities of the intein remained as the unspliced precursors under the experimental conditions. In contrast, the M86 precursors were completely consumed and mostly converted into the splice product with a minor fraction ending up as N-terminal cleavage products (Fig. 6B). 
A
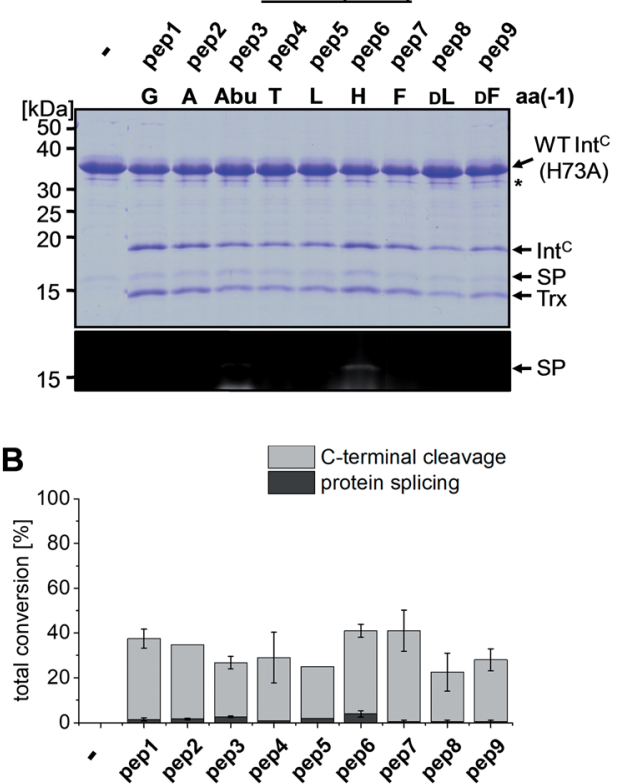
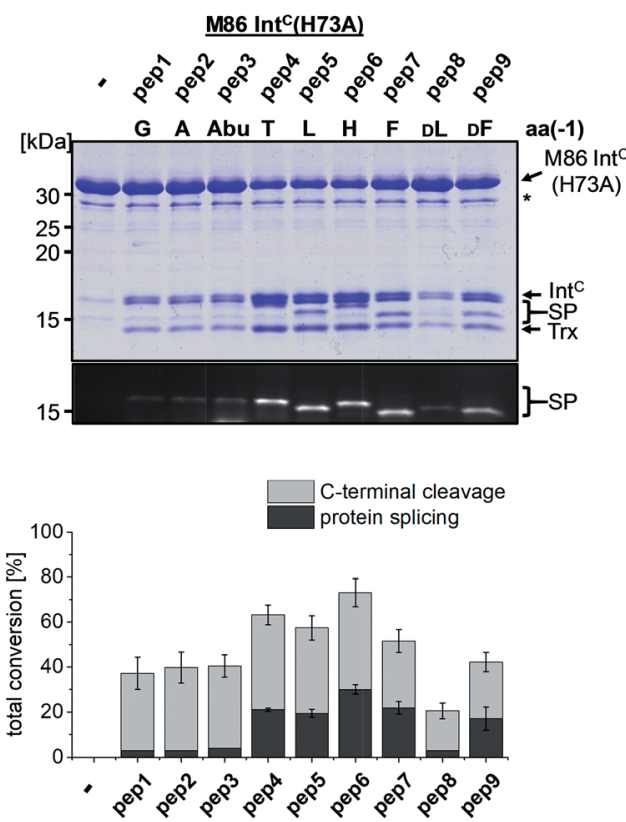

Fig. 5 Extein dependent compensation of the block B histidine in semisynthetic protein trans-splicing. Reaction schemes are as shown in Fig. $1 A$ but using the H73A mutants of both inteins. (A) Analysis of reactions on SDS-PAGE gels using Coomassie-staining (top) or UV illumination (bottom panel). Int ${ }^{C}$ proteins $(20 \mu \mathrm{M})$ were incubated with the indicated peptides $(60 \mu \mathrm{M})$ for $24 \mathrm{~h}$ and then quenched by SDS loading buffer and boiling. The weak bands visible for pep3 in the lower panels seem to result from a slight impurity of this peptide and were also observed in the absence of a protein partner (data not shown). (B) Yields of protein trans-splicing and C-terminal cleavage reactions determined by densitometric analysis of the Coomassie-stained gels shown in A, except for values for pep1-3 and pep8, which were estimated from the fluorescence signals in the bottom panels. Error-bars indicate standard deviations. Asterisks denote a protein contamination.

To address the compensatory mechanism, the cis-inteins with the H73A mutation were prepared with and without combination with the blocked C-terminal splice junction
(H73A, N154A, S+1A = AAA mutants). For both inteins, we observed the same trend in the size dependence of the $(-1)$ residue as found for the split inteins. In the case of the isolated

A
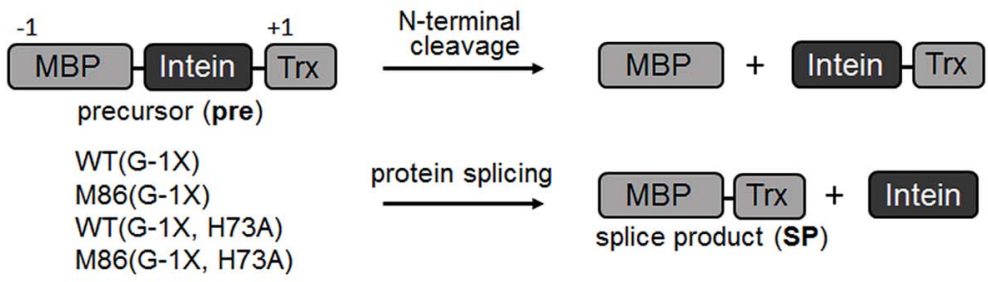

C-terminal cleavage

MBP Intein + Trx

B
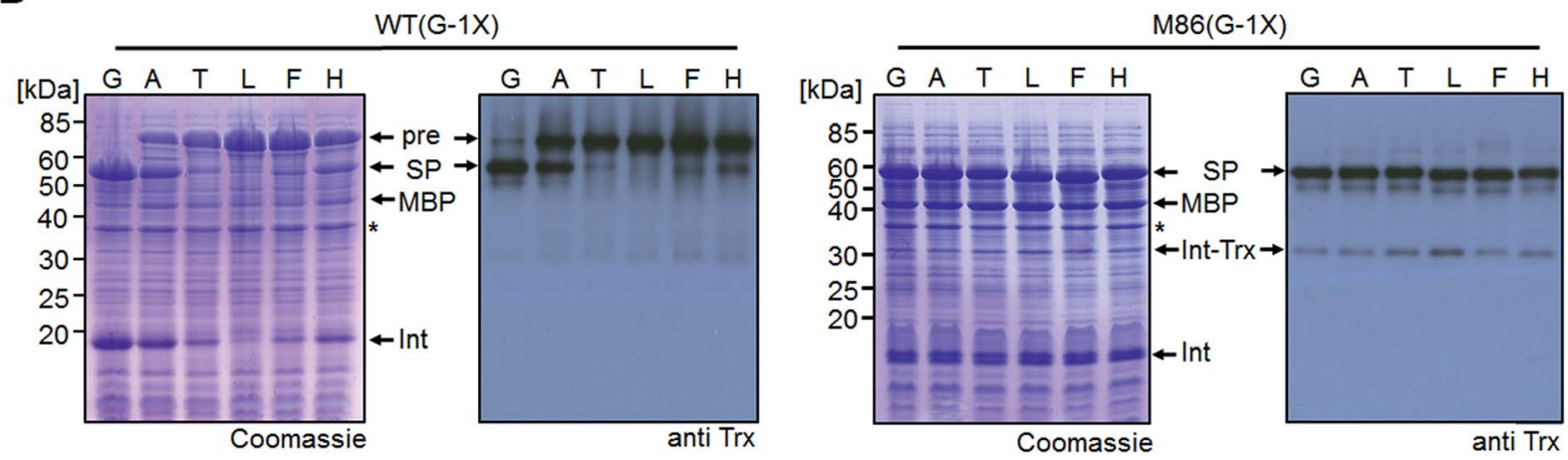

Fig. 6 Extein dependence at the (-1) position of cis-inteins. (A) Reaction schemes. (B) Analysis of reactions on SDS-PAGE gels using Coomassiestaining (left panels) or Western-blotting (right panels). Shown are whole-cell lysates of $E$. coli cells expressing the cis-intein constructs for $3 \mathrm{~h}$ at $37^{\circ} \mathrm{C}$ after induction with IPTG. Aliquots were mixed with SDS loading buffer and boiled. 
$\mathrm{N}-\mathrm{S}$ acyl shift, the precursors remained stable with $\operatorname{Gly}(-1)$, but all $(-1)$ residues larger than alanine supported significant levels of N-terminal cleavage and hence complemented the $\mathrm{N}-\mathrm{S}$ acyl shift (Fig. 7A). Furthermore, when assaying splicing in the context of the respective H73A single mutants, both inteins showed no activity with the native $\operatorname{Gly}(-1)$ extein residue. For the WT intein, larger residues at the $(-1)$ position, in particular His $(-1)$ and $\operatorname{Thr}(-1)$, gave rise to detectable levels of protein splicing and could therefore compensate for the missing His73 residue. This result was in contrast to that of the split WT intein and can likely be explained by a more stable folding of the cisintein that prevents dissociation of the $\mathrm{Ex}^{\mathrm{N}}-\mathrm{Int}^{\mathrm{N}}$ part and helps the intein to stay on-pathway. Similarly, the M86(H73A) mutant spliced with all $(-1)$ substitutions larger than alanine and showed yields up to $\sim 50 \%$ in the case of $\mathrm{Phe}(-1)$ (Fig. 7B). Furthermore, N-terminal cleavage was observed in these cases, suggesting that additional fractions of the proteins underwent the $\mathrm{N}-\mathrm{S}$ acyl shift but then did not stay on-pathway.

\section{Crystal structures reveal different distortions at the N-terminal splice junction}

To investigate the structural consequences of a sterically demanding side chain at the $(-1)$ position, we crystallized the M86 intein as well as its $G(-1) F$ and $G(-1) F / H 73 A$ mutants and determined their structures at $2.0 \AA$, $1.5 \AA$, and $1.2 \AA$ A resolution, respectively (Table $\mathrm{S} 2 \dagger$ ). Similar to the available structure of the
Ssp DnaB mini-intein at $2.0 \AA^{, 14}$ here referred to as the WT intein (PDB entry 1MI8), all three constructs contained the inactivating $\mathrm{C} 1 \mathrm{~A}$ and $\mathrm{N} 154 \mathrm{~A}$ mutations together with five flanking extein amino acids on both sides of the intein. The three variants of M86 crystallized in three different space groups with one or, in the case of the M86 intein itself, two copies of the protein in the asymmetric unit (Table $\mathrm{S} 2 \dagger$ ). Together with the structure of the WT intein, this allows comparing five independent intein monomers.

As expected, all of these monomers show the conserved horseshoe-like fold with nearly identical cores (RMSDs from 0.5-0.6 A, Fig. 8 and Fig. S2†). Major differences only occurred in the flexible, solvent-exposed parts including the $\mathrm{N}$ - and C-terminal extein residues and the loop region covering the site of the deleted homing endonuclease domain. While this region (residue 99-115) was highly flexible in the WT intein and in three of the M86 intein monomers analyzed here, crystal contacts stabilized the respective loop in the $\operatorname{Mr6}(\mathrm{G}(-1) \mathrm{F})$ mutant and allowed tracing. However, comparison to the structured parts of this loop in the other structures indicates that the flexibility of this region leads to principally different conformations, precluding further discussion (Fig. S2 and S3 $\dagger$ ).

Notably, the structures of the WT intein and of M86 as well as of its mutants are overall highly similar (RMSD $=0.6 \AA$ ), suggesting that the differences in the splicing activity must be rooted in subtle structural alterations that can only be identified after careful analysis of local geometric parameters. Since the

A
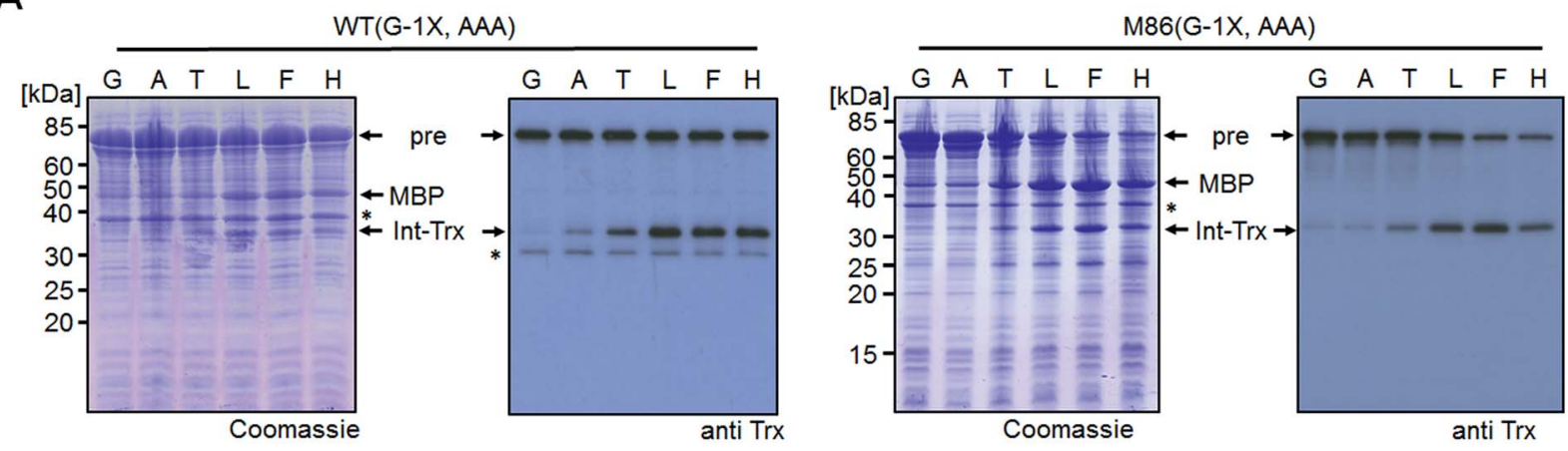

B
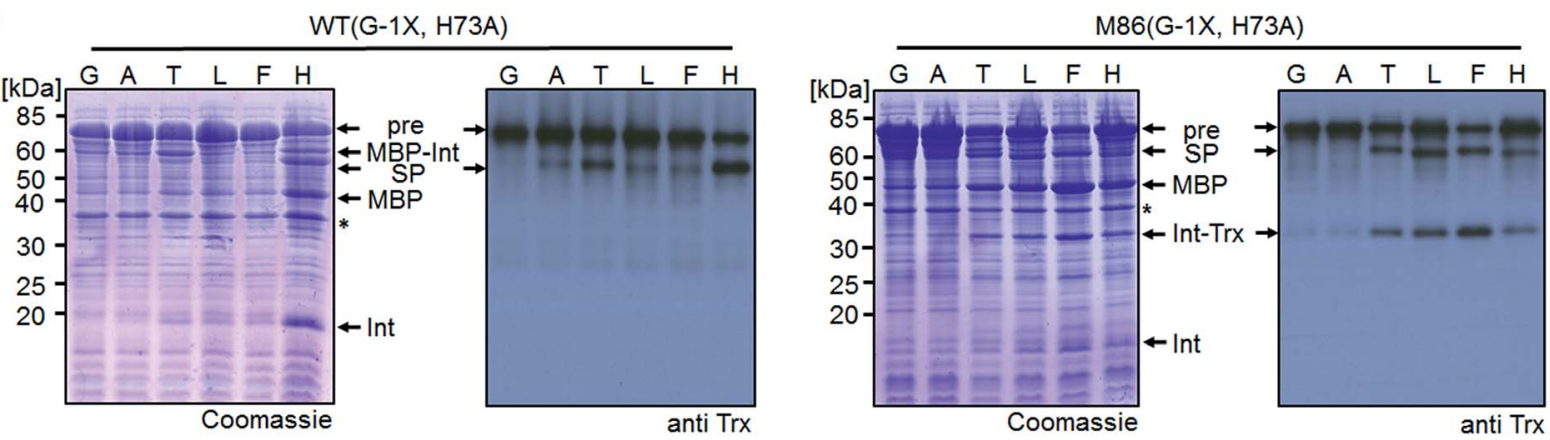

Fig. 7 Extein dependent compensation of block B histidine of cis-inteins. Reaction schemes are as shown in Fig. 5A but using the H73A/N154A/ $\mathrm{S}+1 \mathrm{~A}$ mutant for the isolated $\mathrm{N}-\mathrm{S}$ acyl shift resulting in $\mathrm{N}$-terminal cleavage (panel A) and H73A mutants to allow for protein splicing (panel B), respectively, when the essential histidine residue is compensated for. Reactions were analyzed on SDS-PAGE gels using Coomassie-staining (left panels) or western-blotting (right panels) using whole-cell lysates of $E$. coli cells expressing the cis-intein constructs for $3 \mathrm{~h}$ at $37^{\circ} \mathrm{C}$ after induction with IPTG. Aliquots were mixed with SDS loading buffer and boiled. 

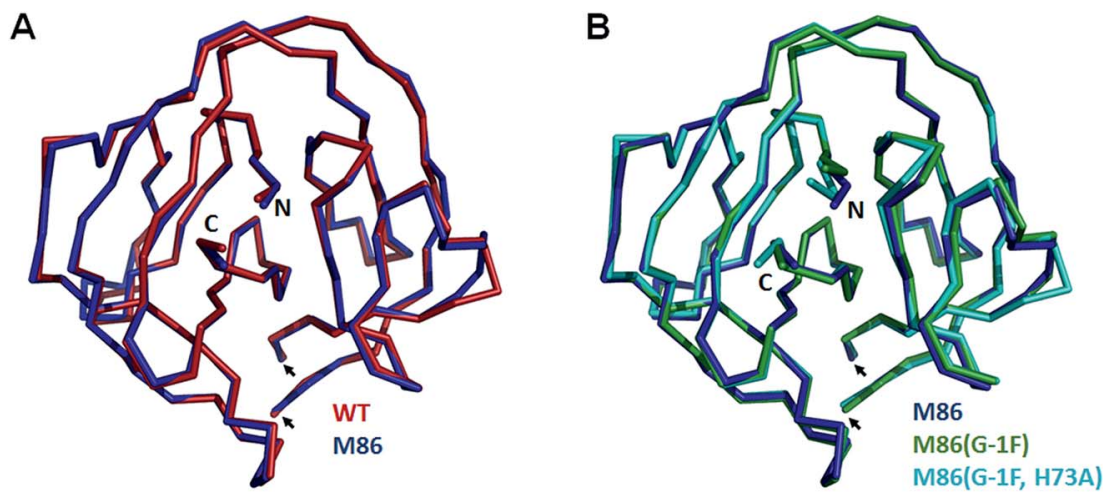

Fig. 8 Overlay of peptide backbones of intein crystal structures. (A) Overlay of the WT Ssp DnaB ${ }^{14}$ and the M86 intein precursors. (B) Overlay of the M86 intein with its extein(-1) and active site mutants. The endonuclease loop regions between amino acids 98 and 116 (indicated with arrows) and extein residues upstream of aa $(-2)$ and downstream of aa $(+2)$ have been omitted for comparability. These regions are illustrated in Fig. S2.广

splice site is located close to the surface of the protein and resides in different crystallographic environments, an influence of crystal contacts in the vicinity of the scissile bond would be conceivable in principle. Residues of the splice site itself are, however, not directly involved in these crystal contacts, because the five additional extein residues at both termini act as spacers that shield the splice site from neighboring molecules (Fig. S3 $\uparrow$ ). It is therefore unlikely that the following observations are a consequence of different crystallographic environments.

When the WT and M86 intein are compared, slight changes of the backbone $\varphi$-, $\psi$ - and $\omega$-angles are observed at the $\mathrm{N}$-terminal splice junction, which may correlate with the altered activity of the M86 mutant. The carbonyl group of the scissile peptide bond is slightly shifted between the two structures, a finding that is also reflected by differences in the $\omega$-angle (Table 2). This angle is closer to $180^{\circ}$ of the perfect trans conformation for the M86 intein. It is, however, unclear how significant these differences are and how they can be interpreted to explain the activity differences between the WT and M86 intein.

Comparison of the M86(G(-1)F) and M86(G(-1)F/H73A) structures with that of the M86 intein, on the other hand, revealed a few striking and obviously more significant changes at the N-terminal splice junction (Fig. 9). The sterically demanding Phe side chain in the $\operatorname{M86}(\mathrm{G}(-1) \mathrm{F})$ mutant is partially squeezed between the His73 imidazole side chain and the N-terminal scissile bond, pushing both moieties slightly apart. The distance between the imidazole ring of the histidine

Table 2 Angles at the $\mathrm{N}$-terminal scissile bond in the four DnaB intein crystal structures

\begin{tabular}{|c|c|c|c|c|c|c|}
\hline \multirow[b]{2}{*}{ Intein } & \multirow[b]{2}{*}{ Pdb-code } & \multicolumn{2}{|c|}{$\mathrm{Aa}(-1)$} & \multirow[b]{2}{*}{$\omega$} & \multicolumn{2}{|l|}{$\operatorname{Ala}(1)$} \\
\hline & & $\phi$ & $\Psi$ & & $\Phi$ & $\Psi$ \\
\hline WT Ssp DnaB & 1MI8 (ref. 14) & $-82^{\circ}$ & $-154^{\circ}$ & $174^{\circ}$ & $173^{\circ}$ & $151^{\circ}$ \\
\hline M86 (chain A) & $6 \mathrm{FRH}$ & $-102^{\circ}$ & $-176^{\circ}$ & $179^{\circ}$ & $-157^{\circ}$ & $159^{\circ}$ \\
\hline M86 (Chain B) & & $-101^{\circ}$ & $-177^{\circ}$ & $179^{\circ}$ & $-158^{\circ}$ & $159^{\circ}$ \\
\hline M86(G-1F) & 6FRG & $-125^{\circ}$ & $168^{\circ}$ & $-173^{\circ}$ & $-167^{\circ}$ & $172^{\circ}$ \\
\hline M86(G-1F, H73A) & 6FRE & $-124^{\circ}$ & $52^{\circ}$ & $-159^{\circ}$ & $-76^{\circ}$ & $154^{\circ}$ \\
\hline
\end{tabular}

side chain and the amide nitrogen of Ala1 increased from $2.9 \AA$ in the M86 structure (with the $\pi$-nitrogen as reference point) to 3.2 or $4.3 \AA$ in the $\mathrm{G}(-1) \mathrm{F}$ mutant ( $\delta$-carbon as reference point), for which two very similar His73 conformers can be observed, indicating increased mobility of this residue (Fig. 9B). This finding suggests that the polar interaction between the $\delta$-nitrogen of the histidine and the scissile bond amide has been disrupted, at least partially, potentially resulting in a loss of the catalytic contribution of the histidine side chain in case of the M86(G(-1)F) mutant. Consistent with this interpretation is a reorientation of the His73 imidazole ring, which is flipped in both conformers of the $\mathrm{G}(-1) \mathrm{F}$ mutant, such that its $\delta$-nitrogen is not facing the amide nitrogen anymore (Fig. 9B). Instead, the flipped imidazole ring of one of the His73 conformers is stabilized directly by the key Asp136 residue of the block F motif, which also displays a rotated side chain relative to the M86 structure and is within the H-bonding distance of $2.9 \AA$ to the imidazole ring. The flipped orientation of the other His73 conformer in the $\mathrm{G}(-1) \mathrm{F}$ mutant is assisted by a water molecule (water 145) that is not present in the M86 structure and establishes hydrogen bonds to both the Asp136 side chain and the imidazole moiety (Fig. 9B). Importantly, the $\mathrm{G}(-1) \mathrm{F}$ mutation also leads to changes within the backbone geometry around the scissile peptide bond, which expresses itself in changes of the dihedral $\varphi$-, $\psi$ - and $\omega$-angles between the $(-1)$ position and Ala1 (Table 2). In the M86(G(-1)F) mutant, the $\omega$-angle $\left(-173^{\circ}\right)$ shows a comparable deviation from the perfect $180^{\circ}$ as observed for the WT intein $\left(174^{\circ}\right)$, albeit with an inverted plane. The effects are even more pronounced in the M86(G(-1)F/H73A) mutant (Table 2 and Fig. 9C). Here, the dihedral angle $\varphi$ of the Ala1 residue is twisted to $-76^{\circ}$, which represents a marked change of up to $91^{\circ}$ relative to the other M86 structures. This angle is typically $\sim 130-170^{\circ}$ in the other intein structures that have been crystallized with flanking $\mathrm{Ex}^{\mathrm{N}}$ residues (Table S3†). The resulting twist rotates the carbonyl of the scissile peptide bond toward the space liberated by the missing His73 side chain. The new space has also accommodated a water molecule (water 5), which is not present in the other two structures. This water molecule seems to stabilize the twisted conformation by 

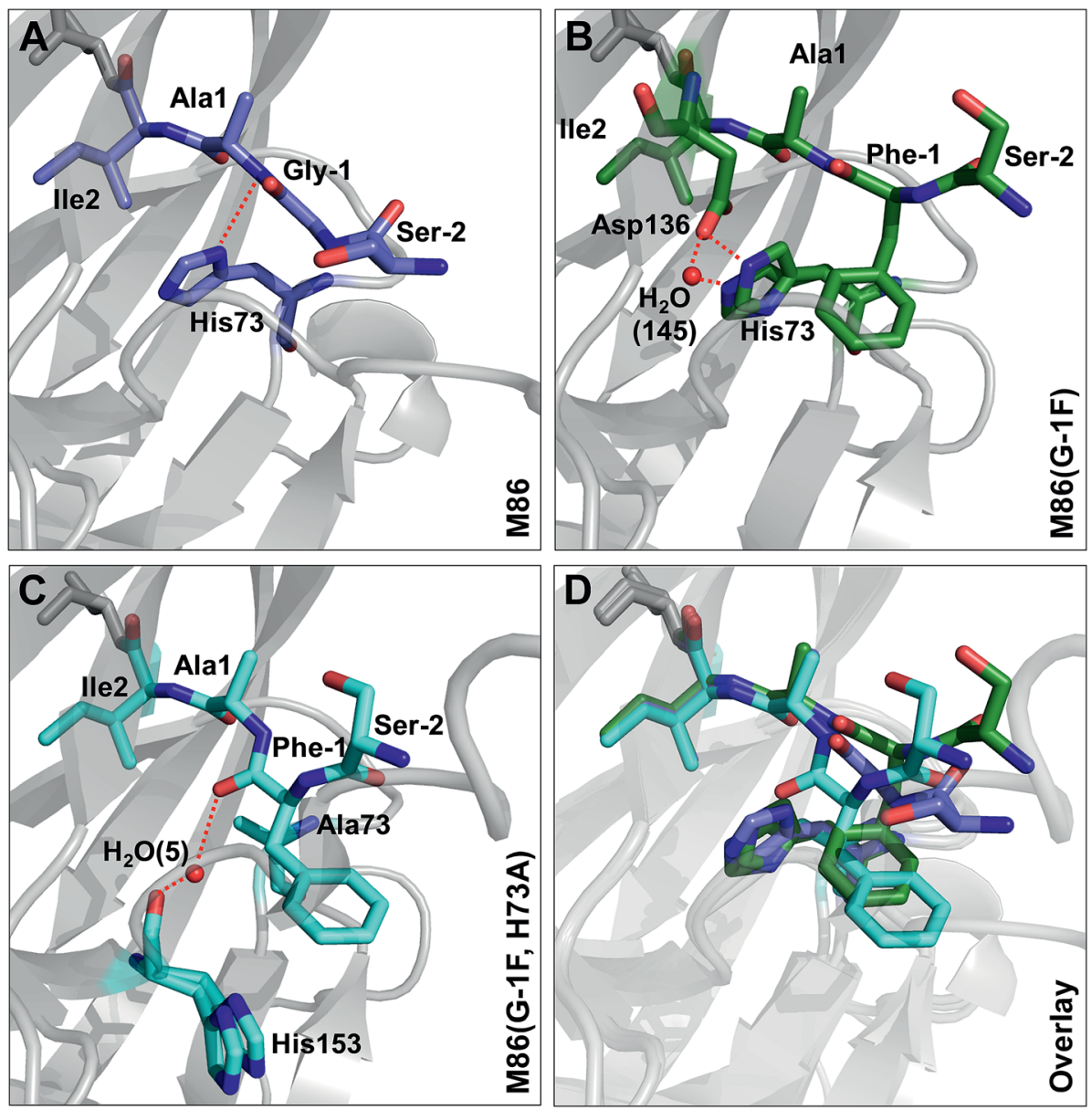

Fig. 9 Effect of mutations on the structure of the $\mathrm{N}$-terminal splice junction. Shown are close-ups of two extein residues upstream and two intein residues downstream of the scissile peptide bond. Panel $\mathrm{C}$ additionally shows a stick representation of the block $\mathrm{G}$ histidine (H153).

coordinating the carbonyl oxygens of Phe $(-1)$ and of the block G His153 (Fig. 9C). Moreover, the $\psi$-angle of the Phe(-1) residue is very unusual with $52^{\circ}$. The overall distortion of the plane with the scissile peptide bond, described by the angle $\omega$ (Table 2), increases from the M86 intein $\left(\omega=179^{\circ}\right)$ to the $\mathrm{G}(-1) \mathrm{F}$ mutant $\left(\omega=-173^{\circ}\right)$ and the $\mathrm{G}(-1) \mathrm{F} / \mathrm{H} 73 \mathrm{~A}$ double mutant $\left(\omega=-159^{\circ}\right)$. In particular the latter structure, with $21^{\circ}$ off from the optimal trans-conformation, suggests that the mutations have a significant effect on the stability of the peptide bond.

\section{Conclusions}

The dependence of inteins on their host sequence is a parameter of great importance for virtually all intein-based applications. In general, the origin of intein dependence on certain flanking amino acids is not well understood and the engineering of inteins with greater or unlimited tolerance is challenging..$^{24,32-34}$ The M86 mutant of the Ssp DnaB intein (here referred to as WT intein) was selected by sequential directed evolution for its more general activity and is one of the few known intein mutants with improved extein promiscuity. ${ }^{24,27}$ This study reports the first systematic investigation on various residues at the upstream flanking $(-1)$ position, which naturally is a Gly residue, of both the M86 mutant and the parent intein. Our data confirmed the general activity of the M86 intein and its clear superiority over the parent intein. Importantly, whereas the WT intein showed no or only very little activity with residues larger than Ala, the M86 mutant was able to splice efficiently with all tested amino acids, both in cis and in trans.

While it has already been well-established that the nature of the flanking extein amino acids can influence the efficiency of the protein splicing reaction, there is little precedent for nonnative extein residues to promote, instead of to impair, the protein splicing pathway. ${ }^{35,36}$ Furthermore, to our knowledge, no such case has been reported in which the loss of a catalytically critical residue has been compensated for by extein optimization, which would indicate a direct functional contribution. As a surprising finding during this study we discovered that substitutions at the $(-1)$ residue enabled both the WT and the M86 intein to catalyze the N-S acyl shift in the context of a H73A/N154A/S+1A mutant, which was designed to block all steps of the splicing pathway. The histidine of the block B motif is essential for this first step in the splicing pathway in the native sequence context (Fig. 1C). The functional complementation of the histidine correlated with the size of the $(-1)$ side chain (Fig. 1D) and became significant with side chains larger 
than Ala or Abu. While it is conceivable for several of our selected amino acids to help catalyze the N-S acyl shift through their side chain, e.g. Thr, His and Phe, the aliphatic Leu side chain also promoted this effect, thus ruling out the requirement of electronic effects. The importance of steric demand was confirmed by the crystal structure of the M86 precursor intein with the $G(-1) F$ mutation and revealed a constraint imposed around the scissile bond. The increased distance of the His73 side chain from the scissile bond indicated that the steric demand at the $(-1)$ position may uncouple His73 from its role in the natural mechanism of the $\mathrm{N}-\mathrm{S}$ acyl shift, at least in part. Strikingly, the functional complementation of the catalytic His73 through a large $(-1)$ side chain also extended to a single H73A mutant of the M86 intein. Here, splicing activity was restored, whereas the same intein with the native $\operatorname{Gly}(-1)$ was completely inactive. This finding showed that the subsequent steps in the protein splicing pathway are not critically impaired, neither by introducing a larger amino acid at position $(-1)$ nor by substituting the catalytic His73, suggesting only local perturbations that do not affect the remaining configuration of the active site. Again, this hypothesis was confirmed by the crystal structure of the $\mathrm{G}(-1) \mathrm{F} / \mathrm{H} 73 \mathrm{~A}$ mutant, which revealed that significant changes are indeed restricted to the $\mathrm{N}$-terminal splice junction. The functional complementation of His73 by large $(-1)$ side chains can be seen as evidence to rule out the critical role of this block B residue in deprotonating Cys1 or in other steps of the pathway; however, an increased ground-state destabilization by the $\mathrm{G}(-1) \mathrm{F}$ mutations might also obviate the need for His73 as a general base. These conclusions are in agreement with an earlier study in which we showed that a single $N$-methyl group at the catalytic Cys1 could also functionally compensate for a mutated His73 side chain. ${ }^{10}$ Thus, in addition to the different natural mechanisms to promote the $\mathrm{N}-\mathrm{S}$ acyl shift that have been recognized so far (see Introduction), these two engineered compensatory mechanisms for the $\mathrm{N}-\mathrm{S}$ acyl shift further underline the functional plasticity of the active site and support the idea that many different catalytic solutions can serve in protein splicing.

Our crystal structures also support the model that the first reaction in protein splicing is largely brought about by groundstate destabilization through distortion around the scissile peptide bond. The structures of the M86(G(-1)F) and M86(G(-1) $\mathrm{F} / \mathrm{H} 73 \mathrm{~A})$ mutants revealed snapshots in which the $(-1)$ and 1 residues adopt unusual geometries (Table 2). The significant differences at the N-terminal splice junction in these two structures indicate that the $\mathrm{N}-\mathrm{S}$ acyl shift can be brought about by different, non-native distortion strategies. A recent study used DFT calculations to underline the fact that the rate of the $\mathrm{N}-\mathrm{S}$ acyl shift is favored in the cis over the trans conformation of the scissile bond in a model amide because of the easier approach of the nucleophile to the carbonyl group. ${ }^{37}$ However, the situation in the intein's active site is more complicated because residues around the scissile bond will shape the conformation and polarization of the peptide chain, participating bonds and groups through steric and electronic contacts. Therefore, the optimal conformation for reaching the transition state may not be possible. We assume that at least one more type of conformational distortion, in addition to those approached by our crystal structures, can support non-native compensatory mechanisms, since our data showed that D-amino acids at the $(-1)$ position compensated for the block $\mathrm{B}$ histidine as well (Fig. 1D). A diastereoisomer at the N-terminal splice junction would be expected to have a different influence along these lines. ${ }^{38}$ Thus, the scaffold of the inteins' active site appears to provide a confined space in which strains beneficial to the $\mathrm{N}-\mathrm{S}$ acyl shift can be exerted on the scissile bond. This space displays functional plasticity for different catalytic solutions. An important residue to hold the peptide in this space seems to be the conserved and well-characterized Thr from the block B motif, which was described to spring-load the N-terminal splice junction in the $S s p$ DnaE intein (corresponding to Thr70 in the WT and M86 variants of the $S s p$ DnaB intein). ${ }^{39}$ Indeed, the position of this active site residue is virtually identical for all four structures compared here (not shown). Furthermore, additional catalytic residues such as the $(+1)$ nucleophilic side chain serve to remove the (thio)ester from the equilibrium through the subsequent steps of the protein splicing pathway.

Notably, large side chains at the $(-1)$ positions do occur in many native inteins. ${ }^{40}$ It is therefore tempting to speculate that the mechanism discovered here also contributes or is even crucial to catalysis in some of those cases. Examples from the literature that may hint at this possibility are the Pho RadA intein and the Tko CDC21-1 intein orthologs. The former intein has a lysine at position $(-1)$ and lower levels of splice product were observed when this residue was replaced with smaller amino acids such as alanine or glycine. ${ }^{34}$ The latter constitute a small group of inteins naturally lacking the block $\mathrm{B}$ histidine residue and were found to have evolved a compensatory mechanism to better stabilize the oxyanion; ${ }^{16}$ however, they also come with a bulky leucine at position (-1). Such inteins could have originated from a parent intein in which the histidine was accidentally lost by spontaneous mutation. They could have remained sufficiently active due to the compensatory mechanism we describe here, followed by further optimization of the alternative catalytic strategy during evolution. Our results suggest this to be a viable possibility for protein engineering attempts, in particular to adapt inteins to very large, possibly unnatural flanking moieties, although it is at present unpredictable which inteins would be favorably amenable to such an approach and what its limitations would be.

In summary, we have further investigated the high sequence promiscuity of the M86 intein and reported its crystal structure. We have discovered and structurally corroborated a new exteindependent mechanism to promote the N-S acyl shift through distortion around the scissile peptide bond independent of the block B histidine that might also be used by native inteins. These results underline the catalytic flexibility of inteins and may be useful for future attempts to engineer inteins with high sequence tolerance.

\section{Experimental section}

\section{Materials and methods}

Synthetic oligonucleotides were ordered from Biolegio. Restriction enzymes were obtained from Fermentas. Buffer 
reagents, antibiotics and media components were purchased from Carl Roth, Applichem, Sigma Aldrich or Thermo Scientific. Ni-NTA agarose was ordered from Cube Biotech. Rabbit-derived anti-Trx antibody was purchased from Sigma-Aldrich and used in $1: 10000$ dilution. Porcine derived anti-rabbit antibody was purchased from Dako and used in $1: 5000$ dilution. Error bars represent standard deviations from at least three independent experiments.

\section{Protein expression and purification}

E. coli BL21 Gold (DE3) cells were transformed with the respective plasmids (Table S4 $\uparrow$ ). Cells were cultured at $37^{\circ} \mathrm{C}$ in LB medium containing the corresponding antibiotics (ampicillin: $100 \mu \mathrm{g} \mathrm{ml} l^{-1}$ or kanamycin: $50 \mu \mathrm{g} \mathrm{ml}^{-1}$ ). Upon reaching an $\mathrm{OD}_{600}$ of $0.6-0.8$, protein expression was induced by IPTG addition $(0.4 \mathrm{mM})$ and carried out for $4 \mathrm{~h}$ at $28^{\circ} \mathrm{C}$. Cells were harvested by centrifugation and the cell pellets were resuspended in the respective purification buffer. All $\mathrm{His}_{6}$-tagged proteins were purified under denaturing conditions $(50 \mathrm{mM}$ Tris/HCl, $300 \mathrm{mM} \mathrm{NaCl}, 8 \mathrm{M}$ urea, pH8). Denaturing purification conditions were not strictly required but yielded higher protein amounts and improved protein purity. Cell lysis under denaturing conditions was performed using a Potter-Elvehjem homogenizer followed by protein purification via nickel affinity chromatography. Protein renaturation was carried out by dialysis against splice buffer $(50 \mathrm{mM}$ Tris/HCl, $300 \mathrm{mM} \mathrm{NaCl}$, 1 mM EDTA, pH7). For protein crystallization, cells were lysed under native conditions using an emulsifier (Avestin EmulsiFlex®-C5 high-pressure emulsifier). Purification was performed via a C-terminal Ssp GyrB(1-150)-CBD-Tag, which allowed purification via chitin affinity chromatography followed by DTT induced Tag removal. ${ }^{10,41}$ Protein concentration was determined via absorption spectroscopy at $280 \mathrm{~nm}$ using the calculated extinction coefficients. For crystallization purposes, the M86 (G-1F) and (G-1F, H73A) mutant inteins were subjected to an additional size exclusion chromatography to separate aggregates. The proteins were loaded onto a HiLoad ${ }^{\mathrm{TM}} 75$ 26/600 Superdex ${ }^{\mathrm{TM}}$ (GE Healthcare) column connected to an ÄKTA purifier FPLC-system (GE Healthcare) and eluted with $50 \mathrm{mM}$ Tris/HCl, $100 \mathrm{mM} \mathrm{NaCl} \mathrm{pH} \mathrm{7.6.} \mathrm{Fractions} \mathrm{containing}$ pure protein were collected, concentrated to $31 \mathrm{mg} \mathrm{ml}^{-1}$, flash frozen in liquid nitrogen and stored at $-80^{\circ} \mathrm{C}$.

\section{Peptide synthesis and purification}

Peptides were synthesized by standard Fmoc solid phase peptide synthesis (SPPS) using a Liberty microwave-assisted peptide synthesizer (CEM, Kamp-Lintford, Germany) and purified by HPLC according to previously published protocols, ${ }^{10,27}$ except for replacing hydroxybenzotriazole (HOBt) with ethyl cyano(hydroxyimino)acetate (Oxyma Pure).

\section{Protein trans-splicing assay}

Protein trans-splicing was performed in splice buffer at $25{ }^{\circ} \mathrm{C}$. The reaction was initiated by mixing the $\mathrm{N}$-terminal and the C-terminal intein fragments at the indicated concentration in the presence of $2 \mathrm{mM}$ TCEP. The reaction was stopped by addition of $4 \times$ SDS-PAGE loading buffer $(500 \mathrm{mM}$ Tris/HCl, $8 \%$ (w/v) SDS, 40\% (v/v) glycerine, 20\% (v/v) $\beta$-mercaptoethanol, $5 \mathrm{mg} \mathrm{L}^{-1}$ bromophenol blue, pH 6.8) and subsequent boiling $\left(10 \mathrm{~min}, 95^{\circ} \mathrm{C}\right)$.

\section{$\mathrm{N}-\mathrm{S}$ acyl shift assay in trans}

Trans-N-S-acyl-shift assays were performed in splice buffer at $25^{\circ} \mathrm{C}$. The reaction was initiated by mixing the $\mathrm{N}$-terminal and the C-terminal intein fragments at the indicated concentration in the presence of $2 \mathrm{mM}$ TCEP or, alternatively, in the presence of $2 \mathrm{mM}$ DTT in the case of HPLC analysis. For SDS-PAGE analysis, the reaction was stopped by addition of $4 \times$ SDSPAGE loading buffer without boiling to keep the intein complex intact. For HPLC analysis, the reaction was stopped by addition of $0.1 \%$ TFA and subsequent boiling $\left(10 \mathrm{~min}, 95^{\circ} \mathrm{C}\right)$ to precipitate protein components. A linear gradient from $10 \%$ to $50 \%$ ACN within 35 min was used (buffer A: $\mathrm{ddH}_{2} \mathrm{O}+0.1 \%$ TFA; buffer B: ACN $+0.1 \%$ TFA; C18 column: Agilent, ZORBAX SBC18 $(50 \times 3 \mathrm{~mm}, 80 \AA$, $1.8 \mu \mathrm{m}))$. Peak identities were determined by ESI/MS.

\section{Protein splicing and N-S acyl shift assay in cis}

E. coli BL21 Gold (DE3) cells harboring the respective expression plasmids were cultured at a $2 \mathrm{ml}$ scale at $37^{\circ} \mathrm{C}$. Upon reaching $\mathrm{OD}_{600} 0.6-0.8$, expression was induced by IPTG $(0.4 \mathrm{mM})$ addition. After $3 \mathrm{~h}$ at $37^{\circ} \mathrm{C}$, the cells were harvested by centrifugation. The cell pellet was resuspended in $\mathrm{dd}_{2} \mathrm{O}$, mixed with $4 \times$ SDS-PAGE loading buffer and boiled $\left(15 \mathrm{~min}, 95{ }^{\circ} \mathrm{C}\right)$ prior to SDS-PAGE analysis.

\section{Protein crystallization}

All crystallization experiments were performed at $20^{\circ} \mathrm{C}$. For the M86 intein, a grid screen based on previously published crystallization conditions for WT Ssp DnaB (PDB-ID: 1MI8) ${ }^{14}$ was performed, using the hanging drop vapor diffusion method. Well-diffracting crystals grew in a $2 \mu \mathrm{l}$ drop consisting of an equal volume of protein $\left(15 \mathrm{mg} \mathrm{ml}{ }^{-1}\right.$ in $20 \mathrm{mM}$ Tris/ $\mathrm{HCl}$, $250 \mathrm{mM} \mathrm{NaCl}, 1 \mathrm{mM}$ EDTA, $\mathrm{pH}$ 8.5) and reservoir solution (0.1 M Tris pH 7.6, 22\% (w/v) PEG4000) equilibrated against $500 \mu \mathrm{l}$ reservoir solution. The crystals were cryoprotected in a reservoir solution supplemented with $20 \%(\mathrm{v} / \mathrm{v})$ glycerol. Crystallization experiments for the M86 (G-1F) and M86 (G-1F, H73A) inteins were carried out with the sitting drop vapor diffusion method. The crystallization drops consisted of $0.2 \mu \mathrm{l}$ of the respective M86 mutant $\left(25 \mathrm{mg} \mathrm{ml}^{-1}\right.$ in $50 \mathrm{mM}$ Tris/HCl $\mathrm{pH} 7.6,100 \mathrm{mM} \mathrm{NaCl}$ ) and an equal volume of reservoir solution and were equilibrated against $60 \mu \mathrm{l}$ reservoir solution. All plates were set up with a Honeybee 961 dispensing robot (Zinsser Analytic). After initial crystallization conditions were identified using the JCSG Core suites I-IV (Qiagen), they were optimized in grid screen format. The reservoir solutions for this were prepared with a Formulator liquid handling system (Formulatrix). Well-diffracting crystals of M86(G-1F) grew after combining the protein with $0.1 \mathrm{M} \mathrm{Na}_{3}$ citrate $\mathrm{pH} 5.3,48 \%(\mathrm{w} / \mathrm{v})$ PEG200. The crystals were already cryoprotected from the precipitant. After grid screen optimization of the M86 (G-1F, 
H73A) mutant, a Silver Bullets ${ }^{\mathrm{TM}}$ (Hampton Research) additive screen was performed to improve the crystal quality. The additive screen was mixed with the optimized precipitant $(0.1 \mathrm{M}$ HEPES pH 7.5, 10\% (w/v) PEG8000) in a ratio of $1: 10$. Finally, well-diffracting crystals were obtained in $0.1 \mathrm{M}$ HEPES $\mathrm{pH} 7.5$, $10 \%(\mathrm{w} / \mathrm{v})$ PEG8000 supplemented with $0.1 \%(\mathrm{w} / \mathrm{v})$ protamine sulfate and $2 \mathrm{mM}$ HEPES pH 6.8. The M86(G-1F, H73A) crystals were cryoprotected supplementing the reservoir solution with $10 \%(\mathrm{v} / \mathrm{v})(2 R, 3 R)-2,3$-butanediol. All crystals were flash-cooled in liquid nitrogen before data collection.

\section{Data collection, processing, phasing and refinement}

Diffraction data were measured at the Swiss Light Source (Paul Scherrer Institute, Villigen, Switzerland) at beamlines X06DA and X10SA at $100 \mathrm{~K}$. The data were integrated and indexed with $\mathrm{XDS}^{\mathbf{4 2}}$ and scaled with Aimless ${ }^{\mathbf{4 3}}$ from the CCP4 suite. ${ }^{\mathbf{4 4}}$ The structures were phased by molecular replacement with Phaser $^{45}$ from the PHENIX suite ${ }^{46}$ using WT Ssp DnaB (PDB-ID: 1MI8) ${ }^{\mathbf{1 4}}$ as the search model. The initial models were refined in iterative cycles of manual refinement with $\mathrm{COOT}^{47}$ and automated refinement with phenix.refine. ${ }^{\mathbf{4 6 , 4 8}}$ The structures were validated with MolProbity ${ }^{49}$ and deposited in the Protein Data Bank ${ }^{50}$ with accession codes 6FRH (M86), 6FRG (M86(G-1F)) and 6FRE (M86(G-1, H73A)). Full data collection and refinement statistics are shown in Table $\mathbf{S} 2 . \dagger$

\section{Conflicts of interest}

There are no conflicts to declare.

\section{Acknowledgements}

We thank the Swiss Light Source (PSI, Villigen) for beamline access and the beamline scientists for their support. The X-ray communities at the MPI Dortmund and the HZI Braunschweig are acknowledged for data collection. This work was funded by the DFG (MO1073/3-2). KF gratefully acknowledges a Ph. D. stipend from the Verband der Chemischen Industrie. M.P. was supported by the HZI Graduate School GS-Fire.

\section{References}

1 R. Hirata, Y. Ohsumk, A. Nakano, H. Kawasaki, K. Suzuki and Y. Anraku, J. Biol. Chem., 1990, 265, 6726-6733.

2 P. M. Kane, C. T. Yamashiro, D. F. Wolczyk, N. Neff, M. Goebl and T. H. Stevens, Science, 1990, 250, 651-657.

3 C. J. Noren, J. Wang and F. B. Perler, Angew. Chem., Int. Ed. Engl., 2000, 39, 450-466.

4 G. Volkmann and H. Iwai, Mol. BioSyst., 2010, 6, 2110-2121. 5 N. H. Shah and T. W. Muir, Chem. Sci., 2014, 5, 446-461.

6 K. Tori, B. Dassa, M. A. Johnson, M. W. Southworth, L. E. Brace, Y. Ishino, S. Pietrokovski and F. B. Perler, J. Biol. Chem., 2010, 285, 2515-2526.

7 S. Pietrokovski, Protein Sci., 1998, 7, 64-71.

8 T. Klabunde, S. Sharma, A. Telenti, W. R. Jacobs Jr and J. C. Sacchettini, Nat. Struct. Biol., 1998, 5, 31-36.
9 A. Romanelli, A. Shekhtman, D. Cowburn and T. W. Muir, Proc. Natl. Acad. Sci. U. S. A., 2004, 101, 6397-6402.

10 J. Binschik and H. D. Mootz, Angew. Chem., Int. Ed. Engl., 2013, 52, 4260-4264.

11 J. I. Mujika and X. Lopez, J. Phys. Chem. B, 2017, 121, 77867796.

12 Z. Du, P. T. Shemella, Y. Liu, S. A. McCallum, B. Pereira, S. K. Nayak, G. Belfort, M. Belfort and C. Wang, J. Am. Chem. Soc., 2009, 131, 11581-11589.

13 A. L. Bachmann and H. D. Mootz, J. Biol. Chem., 2015, 290, 28792-28804.

14 Y. Ding, M. Q. Xu, I. Ghosh, X. Chen, S. Ferrandon, G. Lesage and Z. Rao, J. Biol. Chem., 2003, 278, 3913339142.

15 Z. Du, J. Liu, C. D. Albracht, A. Hsu, W. Chen, M. D. Marieni, K. M. Colelli, J. E. Williams, J. N. Reitter, K. V. Mills and C. Wang, J. Biol. Chem., 2011, 286, 38638-38648.

16 K. Tori, M. Cheriyan, C. S. Pedamallu, M. A. Contreras and F. B. Perler, Biochemistry, 2012, 51, 2496-2505.

17 P. E. Dawson, T. W. Muir, I. Clark-Lewis and S. B. Kent, Science, 1994, 266, 776-779.

18 T. W. Muir, D. Sondhi and P. A. Cole, Proc. Natl. Acad. Sci. U. S. A., 1998, 95, 6705-6710.

19 L. A. Erlich, K. S. Kumar, M. Haj-Yahya, P. E. Dawson and A. Brik, Org. Biomol. Chem., 2010, 8, 2392-2396.

20 D. Macmillan, Synlett, 2017, 28, 1517-1529.

21 O. Melnyk and V. Agouridas, Curr. Opin. Chem. Biol., 2014, 22, 137-145.

22 H. Hojo, Y. Onuma, Y. Akimoto, Y. Nakahara and Y. Nakahara, Tetrahedron Lett., 2007, 48, 25-28.

23 H. Wu, M. Q. Xu and X. Q. Liu, Biochim. Biophys. Acta, 1998, 1387, 422-432.

24 J. H. Appleby-Tagoe, I. V. Thiel, Y. Wang, Y. Wang, H. D. Mootz and X. Q. Liu, J. Biol. Chem., 2011, 286, 34440-34447.

25 W. Sun, J. Yang and X. Q. Liu, J. Biol. Chem., 2004, 279, 35281-35286.

26 C. Ludwig, M. Pfeiff, U. Linne and H. D. Mootz, Angew. Chem., Int. Ed. Engl., 2006, 45, 5218-5221.

27 A. Wasmuth, C. Ludwig and H. D. Mootz, Bioorg. Med. Chem., 2013, 21, 3495-3503.

28 J. K. Bocker, K. Friedel, J. C. Matern, A. L. Bachmann and H. D. Mootz, Angew. Chem., Int. Ed. Engl., 2015, 54, 21162120.

29 S. Palei and H. D. Mootz, ChemBioChem, 2016, 17, 378-382. 30 C. Ludwig, D. Schwarzer and H. D. Mootz, J. Biol. Chem., 2008, 283, 25264-25272.

31 J. C. J. Matern, K. Friedel, J. Binschik, K. S. Becher, Z. Yilmaz and H. D. Mootz, J. Am. Chem. Soc., 2018, 140, 11267-11275.

32 N. H. Shah, E. Eryilmaz, D. Cowburn and T. W. Muir, J. Am. Chem. Soc., 2013, 135, 5839-5847.

33 A. J. Stevens, G. Sekar, N. H. Shah, A. Z. Mostafavi, D. Cowburn and T. W. Muir, Proc. Natl. Acad. Sci. U. S. A., 2017, 114, 8538-8543.

34 J. S. Oeemig, D. Zhou, T. Kajander, A. Wlodawer and H. Iwai, J. Mol. Biol., 2012, 421, 85-99. 
35 M. Cheriyan, S. H. Chan and F. Perler, J. Mol. Biol., 2014, 426, 4018-4029.

36 M. Cheriyan, C. S. Pedamallu, K. Tori and F. Perler, J. Biol. Chem., 2013, 288, 6202-6211.

37 N. K. Devaraj and C. L. Perrin, Chem. Sci., 2018, 9, 17891794.

38 D. Schwarzer, C. Ludwig, I. V. Thiel and H. D. Mootz, Biochemistry, 2012, 51, 233-242.

39 A. K. Dearden, B. Callahan, P. V. Roey, Z. Li, U. Kumar, M. Belfort and S. K. Nayak, Protein Sci., 2013, 22, 557-563.

40 F. B. Perler, Nucleic Acids Res., 2002, 30, 383-384.

41 G. Volkmann and X. Q. Liu, FEBS J., 2011, 278, 3431-3446.

42 W. Kabsch, Acta Crystallogr., Sect. D: Biol. Crystallogr., 2010, 66, 125-132.

43 P. R. Evans and G. N. Murshudov, Acta Crystallogr., Sect. D: Biol. Crystallogr., 2013, 69, 1204-1214.

44 M. D. Winn, C. C. Ballard, K. D. Cowtan, E. J. Dodson, P. Emsley, P. R. Evans, R. M. Keegan, E. B. Krissinel, A. G. Leslie, A. McCoy, S. J. McNicholas, G. N. Murshudov, N. S. Pannu, E. A. Potterton, H. R. Powell, R. J. Read, A. Vagin and K. S. Wilson, Acta Crystallogr., Sect. D: Biol. Crystallogr., 2011, 67, 235-242.
45 A. J. McCoy, R. W. Grosse-Kunstleve, P. D. Adams, M. D. Winn, L. C. Storoni and R. J. Read, J. Appl. Crystallogr., 2007, 40, 658-674.

46 P. D. Adams, P. V. Afonine, G. Bunkoczi, V. B. Chen, I. W. Davis, N. Echols, J. J. Headd, L. W. Hung, G. J. Kapral, R. W. Grosse-Kunstleve, A. J. McCoy, N. W. Moriarty, R. Oeffner, R. J. Read, D. C. Richardson, J. S. Richardson, T. C. Terwilliger and P. H. Zwart, Acta Crystallogr., Sect. D: Biol. Crystallogr., 2010, 66, 213-221.

47 P. Emsley, B. Lohkamp, W. G. Scott and K. Cowtan, Acta Crystallogr., Sect. D: Biol. Crystallogr., 2010, 66, 486-501.

48 P. V. Afonine, R. W. Grosse-Kunstleve, N. Echols, J. J. Headd, N. W. Moriarty, M. Mustyakimov, T. C. Terwilliger, A. Urzhumtsev, P. H. Zwart and P. D. Adams, Acta Crystallogr., Sect. D: Biol. Crystallogr., 2012, 68, 352-367.

49 V. B. Chen, W. B. Arendall 3rd, J. J. Headd, D. A. Keedy, R. M. Immormino, G. J. Kapral, L. W. Murray, J. S. Richardson and D. C. Richardson, Acta Crystallogr., Sect. D: Biol. Crystallogr., 2010, 66, 12-21.

50 H. M. Berman, J. Westbrook, Z. Feng, G. Gilliland, T. N. Bhat, H. Weissig, I. N. Shindyalov and P. E. Bourne, Nucleic Acids Res., 2000, 28, 235-242. 